The University of Southern Mississippi

The Aquila Digital Community

Faculty Publications

$12-1-2012$

\title{
On the Independent Domination Number of Regular Graphs
}

\author{
Wayne Goddard \\ Clemson University, goddard@clemson.edu \\ Michael A. Henning \\ University of Johannesberg, mahenning@uj.ac.za \\ Jeremy Lyle \\ University of Southern Mississippi, sjlyle@olivet.edu \\ Justin Southey \\ University of Johannesburg, just.so@presentable.me
}

Follow this and additional works at: https://aquila.usm.edu/fac_pubs

Part of the Mathematics Commons

\section{Recommended Citation}

Goddard, W., Henning, M. A., Lyle, J., Southey, J. (2012). On the Independent Domination Number of Regular Graphs. Annals of Combinatorics, 16(4), 719-2139.

Available at: https://aquila.usm.edu/fac_pubs/7574

This Article is brought to you for free and open access by The Aquila Digital Community. It has been accepted for inclusion in Faculty Publications by an authorized administrator of The Aquila Digital Community. For more information, please contact Joshua.Cromwell@usm.edu. 


\title{
On the Independent Domination Number of Regular Graphs
}

\author{
Wayne Goddard $^{\mathrm{a}}$, Michael A. Henning ${ }^{\mathrm{b}, 0}$, Jeremy Lyle ${ }^{\mathrm{c}}$, Justin Southey ${ }^{\mathrm{b}}$

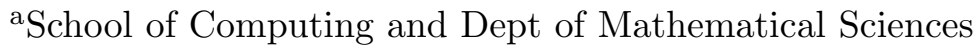 \\ Clemson University, Clemson SC, USA \\ ${ }^{\mathrm{b}}$ Dept of Mathematics, University of Johannesburg \\ Auckland Park, South Africa \\ ${ }^{\mathrm{c}}$ Dept of Mathematics, The University of Southern Mississippi \\ Hattiesburg MS, USA
}

\begin{abstract}
A set $S$ of vertices in a graph $G$ is an independent dominating set of $G$ if $S$ is an independent set and every vertex not in $S$ is adjacent to a vertex in $S$. In this paper, we consider questions about independent domination in regular graphs.

Keywords: Independent domination, regular graph, cubic graph, dominating set

MSC: 05C69, 05C35
\end{abstract}

\section{Introduction}

An independent dominating set in a graph is a set that is both dominating and independent. Equivalently, an independent dominating set is a maximal independent set. Independent dominating sets have been studied extensively in the literature; see for example the books $[16,17]$. In this paper, we consider the independent domination number of regular graphs.

Let $G=(V, E)$ be a graph. A dominating set of $G$ is a set $S$ of vertices of $G$ such that every vertex in $V \backslash S$ is adjacent to a vertex in $S$. The domination number of $G$, denoted by $\gamma(G)$, is the minimum cardinality of a dominating set. An independent dominating set of $G$ is a set that is both dominating and independent in $G$. The independent domination number of $G$, denoted by $i(G)$, is the minimum cardinality of an independent dominating set. The independence number of $G$, denoted by $\alpha(G)$, is the maximum cardinality of an independent set in $G$. In particular,

$$
\gamma(G) \leq i(G) \leq \alpha(G)
$$

\footnotetext{
${ }^{0}$ Research supported in part by the South African National Research Foundation
} 
An independent dominating set of $G$ of cardinality $i(G)$ is called an $i(G)$-set. The concept of independent domination originated in chessboard problems, and elementary properties were provided by Berge [2], while the parameter was defined by Cockayne and Hedetniemi $[5,6]$.

For notation and graph theory terminology we in general follow [17]. Specifically, let $G$ be a graph with vertex set $V$, order $|V|$ and edge set $E$. Let $v$ be a vertex in $V$. The open neighborhood of $v$ is $N(v)=\{u \in V: u v \in E\}$. For a set $S \subseteq V$, its open neighborhood is the set $N(S)=\cup_{v \in S} N(v)$. A vertex of degree 0 is called an isolated vertex. For a set $S \subseteq V$, the subgraph induced by $S$ is denoted by $G[S]$.

Favaron [9] and Gimbel and Vestergaard [10] proved that $i(G) \leq n+$ $2-2 \sqrt{n}$ for a graph $G$ with $n$ vertices and no isolates, and this is sharp. However, this bound is not obtainable for regular graphs, as noted, for example, in [21].

Theorem 1 If $G$ is a regular graph on $n$ vertices with no isolated vertex, then $i(G) \leq \alpha(G) \leq n / 2$.

It is not hard to show that equality is only obtainable for graphs with every component a balanced complete bipartite graph. (This bound is improved upon in Section 4.)

We shall proceed as follows. We focus on 3-regular graphs in Section 2, and on $r$-regular graphs for fixed $r$ in Section 3. Thereafter, in Section 4 we consider regular graphs of large degree, and in Section 5 we consider bounds involving the independent domination numbers of a regular graph and its complement.

\section{Upper Bounds for Cubic Graphs}

The question of best possible bounds for cubic graphs remains unresolved. Lam, Shiu, and Sun [19] provided a proof of the following:

Theorem 2 ([19]) For a connected cubic graph $G$ on $n$ vertices, $i(G) \leq$ $2 n / 5$ except for $K(3,3)$.

Equality in Theorem 2 holds for the prism $C_{5} \times K_{2}$. We believe that the graphs $K(3,3)$ and $C_{5} \times K_{2}$ are the only exception for an upper bound of $3 n / 8$.

Conjecture 1 For a connected cubic graph $G$ on $n$ vertices, $i(G) \leq 3 n / 8$ except for $K(3,3)$ and $C_{5} \times K_{2}$. 
This result would be best possible, as there are two infinite family of cubic graphs with independent domination number three-eighths their order, as we now show. One of these families was also observed in [8].

\subsection{The Families $\mathcal{G}_{\text {cubic }}$ and $\mathcal{H}_{\text {cubic }}$}

The two infinite families $\mathcal{G}_{\text {cubic }}$ and $\mathcal{H}_{\text {cubic }}$ of connected cubic graphs can be constructed as follows.

For $k \geq 1$, define graph $G_{k}$ as described below. Consider two copies of the path $P_{4 k}$ with respective vertex sequences $a_{1} b_{1} c_{1} d_{1} \ldots a_{k} b_{k} c_{k} d_{k}$ and $w_{1} x_{1} y_{1} z_{1} \ldots w_{k} x_{k} y_{k} z_{k}$. For each $1 \leq i \leq k$, join $a_{i}$ to $w_{i}, b_{i}$ to $x_{i}, c_{i}$ to $z_{i}$, and $d_{i}$ to $y_{i}$. To complete $G_{k}$ join $a_{1}$ to $d_{k}$ and $w_{1}$ to $z_{k}$. Let $\mathcal{G}_{\text {cubic }}=\left\{G_{k}\right.$ : $k \geq 1\}$.

For $k \geq 1$, define $H_{k}$ as follows. Consider a copy of the cycle $C_{3 k}$ with vertex sequence $a_{1} b_{1} c_{1} \ldots a_{k} b_{k} c_{k} a_{1}$. For each $1 \leq i \leq k$, add the vertices $\left\{w_{i}, x_{i}, y_{i}, z_{i}^{1}, z_{i}^{2}\right\}$, and join $a_{i}$ to $w_{i}, b_{i}$ to $x_{i}$, and $c_{i}$ to $y_{i}$. To complete the construction of $H_{k}$, for each $1 \leq i \leq k$ and $j \in\{1,2\}$, join $z_{i}^{j}$ to each of the vertices $w_{i}, x_{i}$, and $y_{i}$. Let $\mathcal{H}_{\text {cubic }}=\left\{H_{k}: k \geq 1\right\}$.

Graphs in the families $\mathcal{G}_{\text {cubic }}$ and $\mathcal{H}_{\text {cubic }}$ are illustrated in Figure 1.

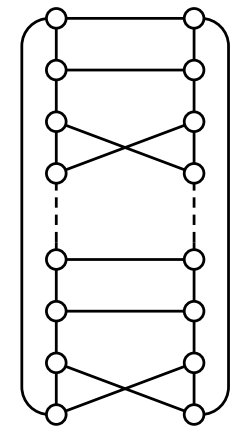

$G_{k}$

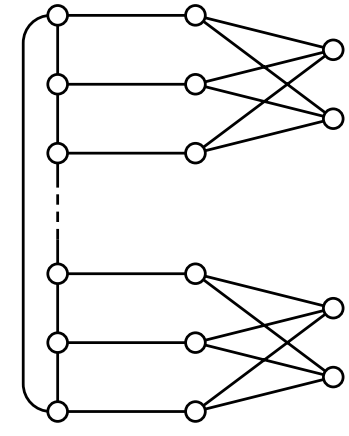

$H_{k}$

Figure 1: Graphs $G_{k}$ and $H_{k}$ of order $n$ with $i\left(G_{k}\right)=i\left(H_{k}\right)=3 n / 8$.

The following result was stated in [8]. It follows from the fact that if $X$ is any independent dominating set in $H_{k} \in \mathcal{H}_{\text {cubic }}$, then $X$ must intersect $\left\{a_{i}, b_{i}, c_{i}, w_{i}, x_{i}, y_{i}, z_{i}^{1}, z_{i}^{2}\right\}$ in at least three vertices.

Proposition 1 If $H_{k} \in \mathcal{H}_{\text {cubic }}$ has order $n$, then $i\left(H_{k}\right)=3 n / 8$.

Proposition 2 If $G_{k} \in \mathcal{G}_{\text {cubic }}$ has order $n$, then $i\left(G_{k}\right)=3 n / 8$. 
Proof. Let $G=G_{k}$ with $n=8 k$. Let $V_{i}=\left\{a_{i}, b_{i}, c_{i}, d_{i}, w_{i}, x_{i}, y_{i}, z_{i}\right\}$ for $i \in[k]=\{1,2, \ldots, k\}$. The set $S=\bigcup_{i=1}^{k}\left\{b_{i}, d_{i}, z_{i}\right\}$ is an independent dominating set of $G$, and so $i(G) \leq|S|=3 k=3 n / 8$.

Let $X$ be an $i(G)$-set, and for $i \in[k]$, let $X_{i}=V_{i} \cap X$. In order to dominate $\left\{b_{i}, c_{i}, x_{i}, y_{i}\right\}$, we note that $\left|X_{i}\right| \geq 2$. Suppose that $|X|<3 n / 8$. Then $\left|X_{i}\right|=2$ for some $i \in[k]$. Let $I_{X}=\left\{1 \leq i \leq k:\left|X_{i}\right|=2\right\}$. Assume that among all $i(G)$-sets, $X$ has been chosen such that $\left|I_{X}\right|$ is minimum. Renaming vertices of $G$, if necessary, we may assume that $\left|X_{k}\right|=2$. We proceed further with the following claim.

Claim: If $\left\{d_{i}, z_{i}\right\} \subseteq X_{i}$ for some $i \in[k]$, then $\left|X_{i}\right|=3$ or $\left|X_{i}\right|=4$. Further, if $\left|X_{i}\right|=3$, then either $a_{i}$ or $w_{i}$ is not dominated by $X_{i}$.

Proof of Claim. If $\left\{a_{i}, w_{i}\right\} \cap X_{i} \neq \emptyset$, then either $a_{i} \in X_{i}$, in which case $x_{i} \in X_{i}$ in order to dominate $x_{i}$, or $w_{i} \in X_{i}$, in which case $b_{i} \in X_{i}$ in order to dominate $b_{i}$. In both cases, $\left|X_{i}\right|=4$. On the other hand, if $\left\{a_{i}, w_{i}\right\} \cap X_{i}=\emptyset$, then either $b_{i} \in X_{i}$, in which case $w_{i}$ is not dominated by $X_{i}$, or $c_{i} \in X_{i}$, in which case $a_{i}$ is not dominated by $X_{i}$. QED

We now consider $G\left[V_{k}\right]$. By our earlier assumption, $\left|X_{k}\right|=2$. In order to dominate $\left\{b_{k}, c_{k}, x_{k}, y_{k}\right\}$, we may assume, by symmetry, that $X_{k}=\left\{b_{k}, y_{k}\right\}$ or $X_{k}=\left\{b_{k}, z_{k}\right\}$ or $X_{k}=\left\{b_{k}, d_{k}\right\}$ or $X_{k}=\left\{c_{k}, y_{k}\right\}$. In all four cases, the vertex $w_{k}$ is not dominated by $X_{k}$. Further, $X_{k}$ contains at most one of $d_{k}$ and $z_{k}$, and so $\left\{d_{k}, z_{k}\right\} \nsubseteq X_{k}$. In order to dominate the vertex $w_{k}$, we have that $z_{k-1} \in X_{k-1}$, where addition is taken modulo $k$. But then $d_{k-1} \in X_{k-1}$ in order to dominate $d_{k}$. Thus, $\left\{d_{k-1}, z_{k-1}\right\} \subseteq X_{k-1}$. By the claim, either $\left|X_{k-1}\right|=3$ or $\left|X_{k-1}\right|=4$.

Suppose $\left|X_{k-1}\right|=3$. Then by the claim, either $a_{k-1}$ or $w_{k-1}$ is not dominated by $X_{k-1}$. This implies that $\left\{d_{k-2}, z_{k-2}\right\} \subseteq X_{k-2}$. By the claim, either $\left|X_{k-2}\right|=3$ or $\left|X_{k-2}\right|=4$. If $\left|X_{k-2}\right|=3$, then $\left\{d_{k-3}, z_{k-3}\right\} \subseteq X_{k-3}$ and $\left|X_{k-3}\right|=3$ or $\left|X_{k-3}\right|=4$.

Continuing this process, we note that since $X$ is an independent dominating set in $G$ and since $\left\{d_{k}, z_{k}\right\} \nsubseteq X_{k}$, there is a smallest integer $j$ such that $\left\{d_{k-j}, z_{k-j}\right\} \subseteq X_{k-j},\left|X_{k-j}\right|=4$ and $1 \leq j \leq k-1$. Thus either $\left\{a_{k-j}, x_{k-j}\right\} \subseteq X_{k-j}$ or $\left\{b_{k-j}, w_{k-j}\right\} \subseteq X_{k-j}$. If $a_{k-j} \in X_{k-j}$, let $v_{k-j}=a_{k-j}$; otherwise, let $v_{k-j}=w_{k-j}$.

We now define the set $X^{\prime}$ as follows. Let $X_{k-j}^{\prime}=\left\{c_{k-j}, v_{k-j}, y_{k-j}\right\}$. Let $X_{k}^{\prime}=X_{k} \cup\left\{w_{k}\right\}$. If $j \geq 2$, then for $k-j<i<k$, let $X_{i}^{\prime}=\left\{c_{i}, w_{i}, x_{i}\right\}$. If $j \leq k-2$, then for $1 \leq i<k-j$, let $X_{i}^{\prime}=X_{i}$. Then, $\left|X_{k}^{\prime}\right|=\left|X_{k}\right|+1=3$, $\left|X_{k-j}^{\prime}\right|=\left|X_{k-j}\right|-1=3$, and $\left|X_{i}^{\prime}\right|=\left|X_{i}\right|$ for all $i \notin\{k, k-j\}$, and so 


$$
\begin{aligned}
& \left|X^{\prime}\right|=|X| \text { but }\left|I_{X^{\prime}}\right|=\left|I_{X}\right|-1 \text {. Let } \\
& \qquad X^{\prime}=\bigcup_{i=1}^{k} X_{i}^{\prime} .
\end{aligned}
$$

Since the set $X$ is an independent dominating set, by construction so too is the set $X^{\prime}$. Thus, $X^{\prime}$ is an $i(G)$-set with $\left|I_{X^{\prime}}\right|<\left|I_{X}\right|$, contradicting our choice of the set $X$. Consequently, $i(G)=3 n / 8$. QED

Perhaps it is even true that $i(G) \leq 3 n / 8$ for $n>10$, with equality if and only if $G \in \mathcal{G}_{\text {cubic }} \cup \mathcal{H}_{\text {cubic }}$. We remark that computer search has confirmed that this is true when $n \leq 20$.

\subsection{Cubic Graphs of Higher Girth}

Verstraete [23] conjectured the following.

Conjecture 2 ([23]) If $G$ is a cubic graph on $n$ vertices of girth at least 6 , then $i(G) \leq n / 3$.

Duckworth and Wormald [8] constructed an infinite family of graphs of girth 5 with independent domination number one-third their order. They also showed that:

Theorem 3 ([8]) If $G$ is a connected cubic graph on $n$ vertices of girth at least 5 , then $i(G) \leq 3 n / 8+O(1)$.

We remark that the girth-six requirement in the above conjecture is essential, since the Generalized Petersen graph $P_{14}$ shown in Figure 2 of order $n=14$ has independent domination number $i\left(P_{14}\right)=5>n / 3$. It seems that perhaps the graph $P_{14}$ is the only exception when relaxing the girth condition from six to five.

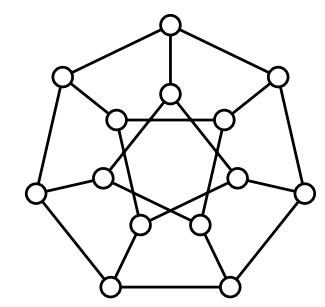

Figure 2: The Generalized Petersen Graph $P_{14}$.

We pose the following conjecture. 
Conjecture 3 If $G \neq K(3,3)$ is a connected bipartite cubic graph on $n$ vertices, then $i(G) \leq 4 n / 11$.

We remark that by computer search we have confirmed that Conjecture 3 is true when $n \leq 26$. If Conjecture 3 is true, then the bound is achieved by the bipartite cubic graph $G$ of order $n=22$ with $i(G)=8$ shown in Figure 3 .

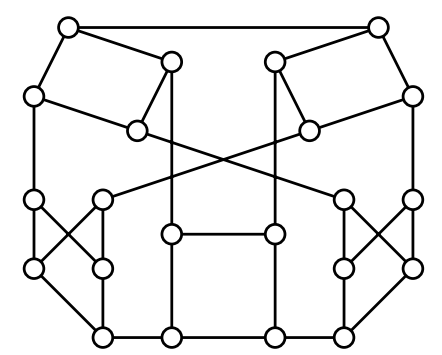

Figure 3: A bipartite cubic graph $G$ with $i(G)=4 n / 11$.

\section{$2.3 \quad i$ versus $\gamma$ in Cubic Graphs}

In 1991, Barefoot, Harary, and Jones [1] gave a class of 2-connected cubic graphs for which the difference between $i$ and $\gamma$ is unbounded and conjectured that for any 3 -connected cubic graph the difference is at most 1 . Their conjecture was disproved in multiple papers, including [7, 18, 24, 25], who showed collectively that there are cubic graphs that are 3 -connected with $\gamma$ and $i$ arbitrarily far apart.

We show now that our family $\mathcal{G}_{\text {cubic }}$ provides a simple example of a family of 3-connected cubic graphs with $\gamma$ and $i$ arbitrarily far apart. Indeed, $i\left(G_{4}\right)=12$ and $\gamma\left(G_{4}\right)=10$, making it the smallest 3-connected cubic graph known with $i-\gamma>1$. In order to prove this fact, we establish the domination number of these graphs.

Proposition 3 If $G \in \mathcal{G}_{\text {cubic }} \cup \mathcal{H}_{\text {cubic }}$ has order $n$, then $\gamma(G)=\lceil 5 n / 16\rceil$.

Proof. Let $G \in \mathcal{G}_{\text {cubic }}$ have order $n=8 k$. If $k=1$, then it is easy to show (and well known that) $\gamma(G)=3=\lceil 5 n / 16\rceil$. So we may assume that $k \geq 2$.

Let

$$
D_{k}=\bigcup_{i=1}^{\lfloor k / 2\rfloor}\left\{a_{2 i-1}, d_{2 i-1}, y_{2 i-1}, x_{2 i}, z_{2 i}\right\} .
$$


If $k$ is even, let $D=D_{k}$, while if $k \geq 3$ is odd, let $D=D_{k} \cup\left\{a_{k}, d_{k}, y_{k}\right\}$. We note that if $k$ is even, then $|D|=5 n / 16$, while if $k$ is odd, then $|D|=$ $5 n / 16+1 / 2$. Since $D$ is a dominating set of $G, \gamma(G) \leq|D|=\lceil 5 n / 16\rceil$. Hence it suffices for us to show that $\gamma(G) \geq\lceil 5 n / 16\rceil$.

Let $[k]=\{1, \ldots, k\}$ and for $i \in[k]$, let $V_{i}=\left\{a_{i}, b_{i}, c_{i}, d_{i}, w_{i}, x_{i}, y_{i}, z_{i}\right\}$. Let $X$ be a dominating set of $G$. For $i \in[k]$, let $X_{i}=X \cap V_{i}$. In order to dominate $\left\{b_{i}, c_{i}, x_{i}, y_{i}\right\}$, it must be that $\left|X_{i}\right| \geq 2$ for all $i \in[k]$. Let $I_{X}=\left\{i \in[k]:\left|X_{i}\right|=\left|X_{i+1}\right|=2\right\}$, where addition is taken modulo $k$, and assume that among all minimum dominating sets, $X$ has been chosen such that $\left|I_{X}\right|$ is minimized.

Suppose that $I_{X} \neq \emptyset$. Renaming indices, if necessary, we may assume for notational convenience that $i=1$. Thus, $\left|X_{1}\right|=\left|X_{2}\right|=2$. By enumerating all possibilities, it follows that $X_{1} \in\left\{\left\{b_{1}, y_{1}\right\},\left\{c_{1}, x_{1}\right\},\left\{c_{1}, y_{1}\right\}\right\}$ and that $X_{2}=\left\{b_{2}, x_{2}\right\}$. In order to dominate $\left\{d_{2}, z_{2}\right\}$, we note that $\left\{a_{3}, w_{3}\right\} \subseteq X_{3}$. Further in order to dominate $\left\{c_{3}, y_{3}\right\}$, we see that $\left|X_{3}\right| \geq 3$. Suppose that $\left|X_{3}\right| \geq 4$. Let $X_{3}^{\prime}=\left\{b_{3}, d_{3}, z_{3}\right\}, X_{2}^{\prime}=\left\{c_{2}, w_{2}, z_{2}\right\}$, and let $X_{i}^{\prime}=X_{i}$ for $i \in$ $[k] \backslash\{2,3\}$. Further let $X^{\prime}=\cup_{i \in[k]} X_{i}^{\prime}$. Then, $X^{\prime}$ is a minimum dominating set with $\left|I_{X^{\prime}}\right|<\left|I_{X}\right|$, a contradiction. Hence, $\left|X_{3}\right|=3$. Thus, either $d_{3} \in X_{3}$ or $z_{3} \in X_{3}$ in order to dominate $\left\{c_{3}, y_{3}\right\}$. By symmetry, we may assume that $z_{3} \in X_{3}$. Then, $a_{4} \in X_{4}$. In order to dominate the set $\left\{c_{4}, x_{4}, y_{4}\right\}$, it follows that $\left|X_{4}\right| \geq 3$. Let $X_{3}^{\prime}=\left\{x_{3}, z_{3}\right\}, X_{2}^{\prime}=\left\{a_{2}, d_{2}, y_{2}\right\}$, and let $X_{i}^{\prime}=X_{i}$ for $i \in[k] \backslash\{2,3\}$. Further let $X^{\prime}=\cup_{i \in[k]} X_{i}^{\prime}$. Then, $X^{\prime}$ is a minimum dominating set with $\left|I_{X^{\prime}}\right|<\left|I_{X}\right|$, once again a contradiction. It follows that $I_{X}=\emptyset$, implying that $\left|X_{i} \cup X_{i+1}\right| \geq 5$ for all $i \in[k]$. This in turn implies that $\gamma(G) \geq\lceil 5 n / 16\rceil$. Consequently, $\gamma(G)=\lceil 5 n / 16\rceil$.

The proof for $H_{k}$ is similar but easier and we omit it. QED

As an immediate consequence of Propositions 2 and 3, we have that if $G \in \mathcal{G}_{\text {cubic }}$ has order $n$, then $i(G)-\gamma(G)=\lfloor n / 16\rfloor$. This suggests the following question:

Question 1 If $G$ is a 3-connected cubic graph on $n \geq 12$ vertices, then is it true that $i(G)-\gamma(G) \leq\lfloor n / 16\rfloor$ ?

We have no idea what the maximum difference is when one considers only connected cubic graphs.

The ratio of the independence number and the domination number in a cubic graph cannot be too large, as is evident from the following result.

Theorem 4 If $G$ is a connected cubic graph, then $i(G) / \gamma(G) \leq 3 / 2$, with equality if and only if $G=K(3,3)$. 
Proof. Let $G=(V, E)$. Among all minimum dominating sets, let $D$ be such that $G[D]$ has the fewest edges. We show that each vertex $v$ of $D$ has at least two neighbors outside $D$. By the minimality of $D$, the vertex $v$ has at least one neighbor outside $D$. Suppose that $v$ has exactly one neighbor $v^{\prime}$ outside $D$. Then $(D \backslash\{v\}) \cup\left\{v^{\prime}\right\}$ is a minimum dominating set that induces a subgraph with fewer edges than $G[D]$, a contradiction. Hence, $v$ has at least two neighbors outside $D$. In particular, $G[D]$ has maximum degree at most 1.

Let $D_{1}$ be a maximum independent set in $G[D]$ and let $D_{2}=D \backslash D_{1}$. Then, $\left|D_{1}\right| \geq|D| / 2$. Let $P_{1}$ be the set of vertices not dominated by $D_{1}$ in $G$. We have $P_{1} \cap D=\emptyset$ and each vertex in $P_{1}$ is adjacent to some vertex of $D_{2}$. Because each vertex of $D_{2}$ has two neighbors outside $D$, we have $\left|P_{1}\right| \leq 2\left|D_{2}\right|$. Let $S_{1}$ be a maximum independent set in $G\left[P_{1}\right]$. Then, $\left|S_{1}\right| \leq\left|P_{1}\right|$ and $D_{1} \cup S_{1}$ is an independent dominating set of $G$. Hence

$$
i(G) \leq\left|D_{1}\right|+\left|S_{1}\right| \leq\left|D_{1}\right|+\left(2|D|-2\left|D_{1}\right|\right)=2|D|-\left|D_{1}\right| \leq 3|D| / 2 .
$$

This establishes the desired upper bound, since $\gamma(G)=|D|$.

Suppose $i(G) / \gamma(G)=3 / 2$. Then we must have equality throughout the Inequality Chain (1). This implies that $D_{1} \cup S_{1}$ is an $i(G)$-set, $\left|S_{1}\right|=\left|P_{1}\right|=$ $2\left|D_{2}\right|$, and $\left|D_{1}\right|=\left|D_{2}\right|=|D| / 2$. Therefore, $P_{1}=S_{1}, N\left(D_{2}\right)=D_{1} \cup P_{1}$, $G[D]=k K_{2}$ for some integer $k \geq 1$, and $N\left(D_{2}\right)$ is an $i(G)$-set. An identical argument shows that $N\left(D_{1}\right)=D_{2} \cup\left(V \backslash\left(N\left(D_{2}\right)\right)\right.$ is an $i(G)$-set. Hence, $G$ is bipartite (with partite sets $N\left(D_{1}\right)$ and $N\left(D_{2}\right)$ ) of order $6 k$, and $i(G)=$ $\left|D_{1}\right|+\left|P_{1}\right|=3 k$. By the discussion after Theorem 1 , this implies that $G=K(3,3)$ QED

We pose the following question.

Question 2 If $G \neq K(3,3)$ is a connected cubic graph, then is it true that $i(G) / \gamma(G) \leq 4 / 3$ ?

\section{Regular Graphs of Fixed Regularity}

Let $c_{r}$ denote the supremum value of $i(G) / n$ taken over all connected $r$ regular graph of order $n$ except $K(r, r)$. By Theorem 1, it follows that $c_{r} \leq 1 / 2$. It is easy to show that $c_{2}=3 / 7$. We saw above that $c_{3}=2 / 5$.

In general, this value is nondecreasing in the sense that $c_{t} \geq c_{r}$ if $r$ is a factor of $t$. To see this, we need the following construction. If $k$ is a positive integer, then the expansion of a graph $G$, denoted by $\exp (G, k)$, is that graph obtained from $G$ by duplicating each vertex $v$ of $G k-1$ times 
to form an independent set $I_{v}$ of size $k$. It is immediate that if $G$ is regular then so is any expansion of $G$. It is also not hard to show (and probably known) that:

Proposition $4 i(\exp (G, k))=k \cdot i(G)$.

As a consequence we obtain:

Lemma 1 For all positive integers $r$ and $s, c_{r s} \geq c_{r}$.

Proof. The expansion of the graphs $G$ that give the value for $c_{r}$ have the same ratio of $i / n$. QED

It follows that $c_{r} \geq 3 / 7$ for all even $r$, attained by the expansions of the 7-cycle. But it is unclear what happens in general. We pose the following questions.

Question 3 Is it true that $c_{r}$ tends to $1 / 2$ as $r \rightarrow \infty$ ?

We pose the following questions for 4-regular graphs.

Question 4 Is $c_{4}=3 / 7$ ? That is, if $G \neq K(4,4)$ is a connected 4-regular graph, then is it true that $i(G) \leq 3 n / 7$ ?

Harutyunyan, Horn, and Verstraete [12] studied independent dominating sets in graphs of girth at least five, and proved the following result.

Theorem 5 ([12]) There is a constant $c>0$ such that for every r-regular graph $G$ on $n$ vertices of girth at least five, $i(G) \leq n(\log r+c) / r$ (where $\log$ denotes the natural logarithm).

\section{1 $i$ versus $\gamma$ in $r$-regular Graphs}

Seifter [22] considered the difference between the domination and independent domination numbers of $r$-regular graphs of connectivity exactly $k$ for all $r \geq 4$. He showed that the difference can be arbitrarily large for all $r \geq 4$ and $1 \leq k \leq r$, except when $r=4$ and $k \in\{1,3\}$. We resolve these two exceptions here.

Lemma 2 There exist 4-regular graphs $G$ of connectivity 1 and connectivity 3 such that $i(G)-\gamma(G)$ is arbitrarily large. 
Proof. We start by noting that the 4-regular expansion of the cycle $C_{m}$ has $\gamma \leq m / 2+1$ and $i=2\lceil m / 3\rceil$. Our goal is to adapt this construction to have the desired connectivity.

For the case of connectivity 1, start with the 2-expansion of a long path. Let $A$ be the four vertices that have degree 2. Then for each $a \in A$, introduce a copy $F_{a}$ of the graph $K_{4,4}-e$, and join $a$ to the two degree-3 vertices in $F_{a}$.

For the case of connectivity 3, start with the 2-expansion of a long path. Let $a_{1}, a_{2}, a_{3}, a_{4}$ be the four vertices that have degree 2 , say with $a_{1}$ and $a_{2}$ having common neighbors. Then add a new vertex $b$ adjacent to all of $a_{1}, a_{2}, a_{3}, a_{4}$, and add edges $a_{1} a_{2}$ and $a_{3} a_{4}$.

In each case the construction produces a 4-regular graph of the desired connectivity, and by making the initial path sufficiently long, the difference $i-\gamma$ can be made arbitrarily large. QED

Question 5 If $G \neq K(4,4)$ is a connected 4-regular graph, then is it true that $i(G) / \gamma(G) \leq 3 / 2$ ?

If Question 5 is true, then the bound is achieved, for example, by the 4-regular expansions of the 7- and 8-cycles, which both have domination number 4 and independent domination number 6 .

\section{Regular Graphs of Large Degree}

In this section we consider the best possible upper bound on $i$ as a function of the order and degree. This bound is hard to pin down, since it is not a continuous function.

Haviland $[13,14]$ provided an upper bound with $n / 4 \leq r \leq n / 2$. We remark that $(3-\sqrt{5}) / 2 \approx 0.3820$.

Theorem 6 ([13, 14]) If $G$ is an r-regular graph on $n$ vertices with $n / 4 \leq$ $r \leq n / 2$, then

$$
i(G) \leq \begin{cases}n-\sqrt{n r}, & \text { if } n / 4 \leq r \leq(3-\sqrt{5}) n / 2 \\ r, & \text { if }(3-\sqrt{5}) n / 2 \leq r \leq n / 2\end{cases}
$$

We will improve on this for $r \geq 2 n / 5$. We will need two previous results. First, we need the lemma of Haviland [13] used in the above proof:

Lemma 3 ([13]) Let $G$ be a graph of order $n$ and minimum degree $\delta$, and let $I$ be an independent dominating set of $G$. If no vertex of $V \backslash I$ is joined to all of $I$, then $i(G) \leq n-\sqrt{n \delta}$. 
Second, we need the following lemma based on an idea in [20]. (It can be extracted from the proof of Lemma 2 in that paper, but since we consider a slightly more general version, we supply the proof.)

Lemma 4 Let $G$ be an r-regular graph on $n$ vertices, and $I_{1}, I_{2}$, and $I_{3}$ be three independent sets of order $t$ such that $I_{1} \cap I_{2} \neq \emptyset$ and $I_{2} \cap I_{3} \neq \emptyset$. If $t>(2 / 3)(n-r)$, then $I_{1} \cap I_{3} \neq \emptyset$.

Proof. Since $I_{1} \cap I_{2} \neq \emptyset$, we may choose a vertex $w \in I_{1} \cap I_{2}$. This implies that $\left|I_{1} \cup I_{2}\right| \leq n-r$, and so $\left|I_{1} \cap I_{2}\right| \geq 2 t-(n-r)$. We can obtain the same bound for $\left|I_{2} \cap I_{3}\right|$. Now, suppose that $I_{1} \cap I_{3}=\emptyset$. Then

$$
\begin{aligned}
\left|I_{2}\right| & \geq\left|I_{1} \cap I_{2}\right|+\left|I_{2} \cap I_{3}\right| \\
& \geq 2(2 t-(n-r)) \\
& =t+3 t-2(n-r) \\
& >t
\end{aligned}
$$

a contradiction. QED

The following result establishes an upper bound for graphs whose complement is connected:

Lemma 5 If $G$ is an $r$-regular graph on $n$ vertices with $r \geq n / 4$, and $\bar{G}$ is connected, then

$$
i(G) \leq(2 / 3)(n-r) .
$$

Proof. Assume $i(G)>(2 / 3)(n-r)$. Since any independent set of order 2 (an edge in the complement) can be extended to an independent set of order $i(G)$, the connectivity of $\bar{G}$ implies that for any two independent sets $I$ and $I^{\prime}$ of order $i(G)$ we can construct a sequence of independent sets $I=I_{1}, I_{2}, \ldots, I_{k}=I^{\prime}$ so that for each $i, I_{i} \cap I_{i+1} \neq \emptyset$ and $\left|I_{i}\right|=i(G)$. Applying Lemma 4, this implies that any two independent sets of order $i(G)$ intersect. In particular, given any $i(G)$-set $I$, there cannot be a vertex $v$ that is adjacent to all of $I$ (since $v$ also lies in some independent set of order $i(G))$. Thus the bound of Lemma 3 applies, but $n-\sqrt{n r} \leq(2 / 3)(n-r)$ in this range. QED

If $G$ is $r$-regular with $r<n / 2$, then its complement is always connected. Thus we obtain:

Theorem 7 If $G$ is an $r$-regular graph on $n$ vertices with $2 n / 5 \leq r<n / 2$, then

$$
i(G) \leq(2 / 3)(n-r)
$$


We note that Theorem 7 (as well as Theorem 6) is sharp when $r=2 n / 5$, as given by the expansion of the 5-cycle, but an accurate determination of the best bounds in the remaining region is still open. One construction is to take the complement of suitable powers of a cycle. Let $G_{n, a}$ be the complement of $C_{n}^{a}$ with $a<n / 4$. (For example, $G_{5,1}$ is $C_{5}$.) This graph has independent domination number $i=a+1$ (achieved by $a+1$ consecutive vertices on the cycle) and is regular of degree $r=n-2 a-1$, so that $i \approx(1 / 2)(n-r)$.

If we consider $r>n / 2$, then upper bounds for this case were determined by Favaron [9].

Theorem 8 ([9]) If $G$ is an $r$-regular graph on $n$ vertices with $r \geq n / 2$, then $i(G) \leq n-r$ with equality attained only by complete multipartite graphs with partite sets all of the same order.

Since the first case where we have equality when $r>n / 2$ is $r=2 n / 3$, there is the question of what happens before then. We have a partial answer:

Theorem 9 If $G$ is an $r$-regular graph with $r>n / 2$, then

$$
i(G) \leq \begin{cases}2(n-r) / 3, & \text { if } r \leq 4 n / 7 \\ r / 2, & \text { if } r \geq 4 n / 7\end{cases}
$$

Proof. Note that at $r=4 n / 7$ the two bounds coincide. We have already shown that if $\bar{G}$ is connected, then $i(G) \leq 2(n-r) / 3$. So it remains to consider the case when $\bar{G}$ is disconnected. If $\bar{G}$ has three or more components, then the smallest component has order at most $r / 2$, and any maximal clique in that forms an independent dominating set in $G$. If $\bar{G}$ has two components, then let $H$ be the larger. Since $H$ has at least $n / 2$ vertices and degree at most $(n-3) / 2$, it follows that $H$ is not complete, and so by Theorem 1 any maximal clique has order at most half the order of $H$, which is at most $r$. That maximal clique forms an independent dominating set in $G$. QED

This bound is sharp at $r=4 n / 7$ : consider the two 4-regular graphs of order 7 and expansions thereof. It is also sharp at $r=2 n / 3$ by the expansions of the triangle. For $r>2 n / 3$, the previous upper bound of $n-r$ is better. 


\section{Nordhaus-Gaddum Bounds}

\subsection{The sum $i(G)+i(\bar{G})$}

For general graphs, the upper bound on the sum is easy: $i(G)+i(\bar{G}) \leq n+1$, (which follows from the bound $i(G) \leq n-\Delta(G)$ ). However, this is only achievable if isolated or dominating vertices are allowed, for instance, if $G=K_{n}$. If such vertices are not allowed, the optimal upper bound is the result from [11]:

Proposition 5 ([11]) For a graph $G$ without isolated or dominating vertices,

$$
i(G)+i(\bar{G}) \leq n+4-\lfloor 2 \sqrt{n}\rfloor,
$$

and this is sharp.

The extremal graphs for the above result are not regular. We conjecture that:

Conjecture 4 For a regular graph $G$ that is neither complete nor empty,

$$
i(G)+i(\bar{G}) \leq n / 2+2 .
$$

If true, the bound in the conjecture is sharp. Consider, for example, a balanced complete bipartite graph, or in general, a regular graph where each component is a balanced complete bipartite graph. Haviland [15] showed that the bound holds if the degree of regularity is at most $n / 4$.

Theorem 10 ([15]) If $G$ is an $r$-regular graph on $n$ vertices with $r<n / 2$, then

$$
i(G)+i(\bar{G}) \leq \begin{cases}n / 2+2, & \text { if } 1 \leq r \leq n / 4, \\ n+2 r-2 \sqrt{n r}+2, & \text { otherwise. }\end{cases}
$$

The best upper bound we can prove is:

Theorem 11 For a regular graph $G$ that is neither complete nor empty,

$$
i(G)+i(\bar{G}) \leq 5 n / 9+2 .
$$

This bound is a direct consequence of Theorem 10 and the following result. 
Theorem 12 If $G$ is an $r$-regular graph on $n$ vertices with $r<n / 2$, then

$$
i(G)+i(\bar{G}) \leq n-r+2 .
$$

Proof. For ease of notation, let $s=i(G)$ and $t=i(\bar{G})$. Choose a maximal clique $T$ with $t$ vertices, and a maximal independent set $S$ with $s$ vertices. Let $S^{\prime} \subset S$ be a set of $s-1$ vertices such that $S^{\prime} \cap T=\emptyset$. Let $w \in S^{\prime}$ be a vertex that minimizes the quantity $|N(w) \cap T|$, and set $T_{w}=N(w) \cap T$. Note that since $T$ is maximal, $\left|T_{w}\right|<t$. Further, if $N(w) \cap T=\emptyset$, then $s+t+r \leq n$, and the result follows; so we may assume otherwise.

We can then use this information to determine two inequalities.

- Consider a vertex $v \in T \backslash T_{w}$ that maximizes the quantity $\left|N(v) \cap S^{\prime}\right|$. Then the set $\{w, v\}$ can be extended to an independent set $S^{\prime \prime}$ of cardinality $s$ that overlaps $S^{\prime}$ in at most $s-1-\left|N(v) \cap S^{\prime}\right|$ vertices. In addition, $w$ is not adjacent to any vertex in $T \backslash T_{w}$. Therefore,

$$
\begin{aligned}
n-r=1+\operatorname{deg}_{\bar{G}}(w) & \geq\left|S^{\prime} \cup S^{\prime \prime}\right|+\left|T \backslash T_{w}\right| \\
& \geq 2 s-1-\left(s-1-\max _{v \in T \backslash T_{w}}\left|N(v) \cap S^{\prime}\right|\right)+t-\left|T_{w}\right| \\
& =s+t-\left|T_{w}\right|+\max _{v \in T \backslash T_{w}}\left|N(v) \cap S^{\prime}\right|
\end{aligned}
$$

- Since $i(\bar{G})=t$, we can expand the clique $\{w\} \cup T_{w}$ to a clique $T^{\prime}$ of order $t$, that overlaps $T$ in exactly $T_{w}$ vertices. Now, consider a vertex $v \in T_{w}$ that maximizes the quantity $\left|N(v) \cap S^{\prime}\right|$. This vertex is adjacent to every vertex in both $T$ and $T^{\prime}$, along with its neighbors in $S^{\prime}$, so that

$$
r \geq 2(t-1)-\left|T_{w}\right|+\max _{v \in T_{w}}\left|N(v) \cap S^{\prime}\right| .
$$

If we multiply inequality (2) by $\left|T \backslash T_{w}\right|$ and inequality (3) by $\left|T_{w}\right|$ and add the resultant expressions, we get

$(n-r)\left(t-\left|T_{w}\right|\right)+r\left|T_{w}\right| \geq\left(t-\left|T_{w}\right|\right)(s+t)+2(t-1)\left|T_{w}\right|-t\left|T_{w}\right|+e\left(S^{\prime}, T\right)$,

where $e\left(S^{\prime}, T\right)$ denotes the number of edges from $S^{\prime}$ to $T$. Since $e\left(S^{\prime}, T\right) \geq$ $(s-1)\left|T_{w}\right|$ by the choice of $w$, this simplifies to

$$
s t+t^{2} \leq n t-r t-(n-2 r)\left|T_{w}\right|+3\left|T_{w}\right| .
$$

This implies

$$
s+t<n-r+3
$$

since $n-2 r>0$. This completes the proof. QED 


\subsection{The product $i(G) \cdot i(\bar{G})$}

Several articles, including [3] and [4], considered the product $i(G) \cdot i(\bar{G})$ for general graphs. In the latter, Cockayne et al. determined that the maximum value of the product is asymptotically $n^{2} / 16$. The asymptotic bound was made precise by the following result from [11]:

Proposition 6 ([11]) Define $b(n)=\lfloor(n+4) / 4\rfloor\lfloor(n+6) / 4\rfloor$. Then, for all graphs $G$ of order $n$,

$$
i(G) \cdot i(\bar{G}) \leq \begin{cases}n & \text { if } n \leq 7 \\ b(n)+1 & \text { if } n=x^{2} \text { for } x \text { odd, or } n=x^{2}-1 \text { for } x \text { even } \\ b(n) & \text { otherwise }\end{cases}
$$

and this is best possible for all $n$.

We do not believe that this bound is even approximately achievable for regular graphs, but are unable to improve on it. Haviland [15] showed that one can improve on the upper bound if one restricts to regular graphs of small degree. We conclude by showing that there are graphs where the product of the two parameters is quadratic in the order, thus answering a question from [15].

Let $x$ be a positive integer. Let $c=9 x$ and $a=18 x-3$. Take two disjoint cliques $A_{1}$ and $A_{2}$ of size $a$ and an independent set $C$ of size $c$. Arrange the vertices of $C$ into 9 cells arranged in a $3 \times 3$ grid, with each cell containing $x$ vertices. Divide $A_{1}$ into three groups of equal size, and join each group to the vertices of a different pair of rows in $C$. Similarly divide $A_{2}$ into three equal groups, and join each group to two columns of $C$. Then the graph $G_{x}$ is regular of degree $24 x-4$ and order $45 x-3$.

Lemma 6 We have $i\left(G_{x}\right)=2+x$ and $i\left(\bar{G}_{x}\right)=12 x-1$. Thus $i \bar{i} / n^{2} \approx$ $1 / 168 \frac{3}{4}$.

Proof. For an independent dominating set of $G_{x}$, start with one vertex from each of $A_{1}$ and $A_{2}$, and this leaves $x$ independent vertices undominated in $C$. For an independent dominating set of $\bar{G}_{x}$, start with one vertex in $C$, and then one can take vertices from only one of $A_{1}$ or $A_{2}$. QED

More generally, one can adjust the construction so that the vertices of $C$ are arranged in a $y \times y$ grid and the vertices of $A_{1}$ and $A_{2}$ are divided into $y$ groups each adjacent to $z$ rows or $z$ columns of $C$. Some calculus shows that best ratio is attained at $z / y \approx 0.6331$, where the ratio $i \bar{i} / n^{2}$ would be 
approximately $1 / 163.5$. Since this is a modest improvement on the above, and it is unclear how close one can get to this ratio in an actual graph, we do not pursue the details.

\section{References}

[1] C. Barefoot, F. Harary, and K.F. Jones. What is the difference between the domination and independent domination numbers of cubic graph? Graphs Combin., 7:205-208, 1991.

[2] C. Berge. Theory of Graphs and its Applications. Methuen, London, 1962.

[3] E.J. Cockayne, O. Favaron, H. Li, and G. MacGillivray. The product of the independent domination numbers of a graph and its complement. Discrete Math., 90:313-317, 1991.

[4] E.J. Cockayne, G. Fricke, and C.M. Mynhardt. On a Nordhaus-Gaddum type problem for independent domination. Discrete Math., 138:199205, 1995.

[5] E.J. Cockayne and S.T. Hedetniemi. Independence graphs. Congr. Numer., X:471-491, 1974.

[6] E.J. Cockayne and S.T. Hedetniemi. Towards a theory of domination in graphs. Networks, 7:247-261, 1977.

[7] E.J. Cockayne and C.M. Mynhardt. Independence and domination in 3-connected cubic graphs. J. Combin. Math. Combin. Comput., 10:173$182,1991$.

[8] W. Duckworth and N. Wormald. Linear programming and the worstcase analysis of greedy algorithms on cubic graphs. Electron. J. Combin., 17(1):Research Paper 177, 28 pp. (electronic), 2010.

[9] O. Favaron. Two relations between the parameters of independence and irredundance. Discrete Math., 70:17-20, 1988.

[10] J. Gimbel and P.D. Vestergaard. Inequalities for total matchings of graphs. Ars Combin., 39:109-119, 1995.

[11] W. Goddard and M.A. Henning. Nordhaus-Gaddum bounds for independent domination. Discrete Math., 268:299-302, 2003. 
[12] A. Harutyunyan, P. Horn, and J. Verstraete. Independent dominating sets in graphs of girth five. manuscript: http://www.math.ucsd.edu/jverstra/indom-final.pdf, 2010.

[13] J. Haviland. On minimum maximal independent sets of a graph. Discrete Math., 94:95-101, 1991.

[14] J. Haviland. Independent domination in regular graphs. Discrete Math., 143:275-280, 1995.

[15] J. Haviland. Upper bounds for independent domination in regular graphs. Discrete Math., 307:2643-2646, 2007.

[16] T.W. Haynes, S.T. Hedetniemi, and P.J. Slater. Domination in Graphs: Advanced Topics. Marcel Dekker Inc., New York, 1998.

[17] T.W. Haynes, S.T. Hedetniemi, and P.J. Slater. Fundamentals of Domination in Graphs. Marcel Dekker Inc., New York, 1998.

[18] A.V. Kostochka. The independent domination number of a cubic 3connected graph can be much larger than its domination number. Graphs Combin., 9:235-237, 1993.

[19] P.C.B. Lam, W.C. Shiu, and L. Sun. On independent domination number of regular graphs. Discrete Math., 202:135-144, 1999.

[20] L. Rabern. On hitting all maximum cliques with an independent set. J. Graph Theory, 66:32-37, 2011.

[21] M. Rosenfeld. Independent sets in regular graphs. Israel J. Math., 2:262-272, 1964.

[22] N. Seifter. Domination and independent domination numbers of graphs. Ars Combin., 38:119-128, 1994.

[23] J. Verstraete. Personal communication. August 2010.

[24] J. Žerovnik and J. Oplerova. A counterexample to conjecture of Barefoot, Harary, and Jones. Graphs Combin., 9:205-207, 1993.

[25] I.È. Zverovich and V.È. Zverovich. Disproof of a conjecture in the domination theory. Graphs Combin., 10:389-396, 1994. 